\title{
Isotope Exchange Experiments on TEXTOR and TORE SUPRA Using Ion Cyclotron Wall Conditioning and Glow Discharge Conditioning
}

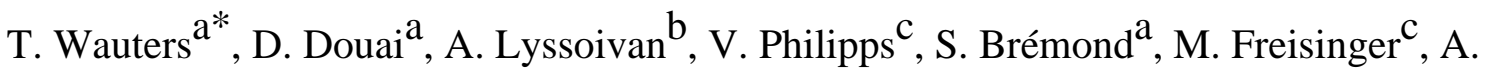 \\ Kreter $^{\mathrm{c}}$, G. Lombard ${ }^{\mathrm{a}}$, O. Marchuk ${ }^{\mathrm{C}}$, P. Mollard ${ }^{\mathrm{a}}$, M.K. Paul ${ }^{\mathrm{b}}$, B. Pegourié ${ }^{\mathrm{a}}$, H. Reimer ${ }^{\mathrm{c}}$, G. \\ Sergienko $^{\mathrm{c}}$, E. Tsitrone ${ }^{\mathrm{a}}$, M. Vervier ${ }^{\mathrm{b}}$, G. Van Wassenhove ${ }^{\mathrm{b}}$, D. Wünderlich ${ }^{\mathrm{d}}$, M. Van \\ Schoor ${ }^{b}$, and G. Van Oost ${ }^{\mathrm{e}}$ \\ ${ }^{a}$ CEA, IRFM, Association Euratom-CEA, 13108 St Paul lez Durance, France.

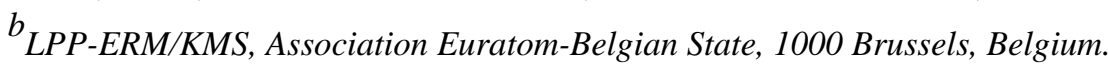 \\ ${ }^{C}$ IEF-Plasmaphysik FZ Jülich, Euratom Association, 52425 Jülich, Germany.

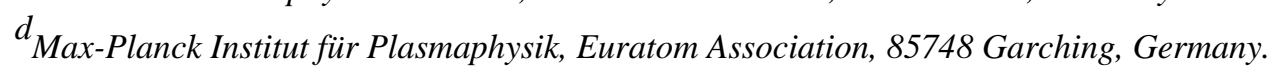 \\ ${ }^{e}$ Ghent University, Department of Applied Physics, 9000 Ghent, Belgium.
}

\begin{abstract}
This contribution reports on isotope exchange studies with both Ion Cyclotron Wall Conditioning (ICWC) and Glow Discharge Conditioning (GDC) in TEXTOR and TORE SUPRA. The discharges have been carried out in $\mathrm{H}_{2}, \mathrm{D}_{2}$ (ICWC and GDC) and $\mathrm{He} / \mathrm{H}_{2}$ mixtures (ICWC). The higher reionization probability in ICWC compared to GDC, following from the 3 to 4 orders of magnitude higher electron density, leads to a lower pumping efficiency of wall desorbed species. GDC has in this analysis (5-10) times higher removal rates of wall desorbed species than ICWC, although the wall release rate is 10 times higher in ICWC. Also the measured high retention during ICWC can be understood as an effect of the high reionization probability. The use of short RF pulses $(\sim 1 s)$ followed by a larger pumping time significantly improves the ratio of implanted over recovered particles, without severely lowering the total amount of removed particles.
\end{abstract}

PACS: 52.27.-h, 52.40.Hf, 52.80.Pi

JNM keywords: Plasma-Materials Interaction, Plasma Properties, Hydrogen and Hydrides PSI-19 keywords: TEXTOR, TORE SUPRA, ITER, Wall conditioning, ICRF

*Corresponding author address: CEA, IRFM, Association Euratom-CEA, 13108 St Paul lez Durance, France.

*Corresponding author E-mail: Tom.Wauters@cea.fr

Presenting author: Tom Wauters

Presenting author e-mail: Tom.Wauters@cea.fr 


\section{$\underline{\text { Introduction }}$}

The almost permanent presence of the high magnetic field in superconducting fusion machines, i.e. ITER, prevents the use of conventional Glow Discharge Conditioning (GDC) for inter-pulse and overnight conditioning. RF plasmas are fully compatible with the presence of a high toroidal magnetic field. For the operation of ITER, Ion Cyclotron Wall Conditioning (ICWC) is envisaged to clean the wall from impurities, to control the wall isotopic ratio and the desorption of hydrogen isotopes $(H, D, T)$. A strong $R \& D$ program with various experiments on different devices is advancing to consolidate this technique and to qualify ICWC for application in ITER and future machines. This paper reports on isotope exchange experiments with ICWC in TEXTOR and TORE SUPRA. During isotope exchange conditioning discharges hydrogen isotopes are desorbed from the wall surface and replaced by another hydrogen isotope. The results of ICWC are compared with isotope exchange experiments using conventional glow discharges on the same machines with typically the same wall pre-treatment conditions. The physics of GDC is well documented [1,2]. The flux of particles to the wall consists mainly of ions $Q_{f t w} \approx 1 \cdot 10^{20} / \mathrm{s}=2.9 \cdot 10^{18} / \mathrm{sm}^{2}$ (TEXTOR) with ion energies of typically $T_{i} \approx 250 \mathrm{eV}$, obtained in the cathode fall in front of the walls. Also an additional contribution of neutrals is not excluded [2]. This paper concentrates on a comparison of measured and deduced quantities in both wall conditioning procedures, without regarding in more detail the dominant Plasma Wall Interaction (PWI) processes at the wall itself. The latter could be omitted in the scope of this paper, however it remains nessecary in a more exstensive comparison between the two techniques.

\section{Isotope exchange with ICWC}

The efficiency of isotopic exchange with ICWC can to a certain extent be deduced from partial pressure measurements obtained from mass spectrometry. Figure 1 shows the hydrogen partial pressures of a $6 \mathrm{~s} \mathrm{He} / \mathrm{H}_{2}$ ICWC discharge in TEXTOR. This discharge is the 
$5^{\text {th }}$ discharge after a wall preloading by $\mathrm{D}_{2}$-GDC, in a series of similar ICWC discharges operated at maximal hydrogen injection with the help of feedback controlled pressure $\left(p_{\text {tot }}=50 \cdot 10^{-3} \mathrm{~Pa}\right)$, overlaid with a constant He flow $\left(p_{\mathrm{He}}=35 \cdot 10^{-3} \mathrm{~Pa}\right)$. The coupled RF power was $50 \mathrm{~kW}$ at an RF frequency of $29 \mathrm{MHz}$ and a toroidal magnetic field of $B_{T}=1.92 \mathrm{~T}$. The figure shows that during the RF discharge the $\mathrm{H}_{2}$ partial pressure is lower than that of $\mathrm{HD}$ and $\mathrm{D}_{2}$. Since the only source of deuterium in the vessel is the wall it is can concluded that during this discharge the wall is acting as the major particle source.

\section{Estimation of wall release rate}

The magnitude of this wall source follows from the balance equation for neutral molecules in the vessel, in a 0D approach which is valid in case the mean free path of the molecules is larger than the dimensions of the vessel:

$$
\frac{d p_{H 2}}{d t}=\frac{Q_{S v, H 2}}{V}+\frac{Q_{S w, H 2}}{V}-\frac{p_{H 2} S}{V}-\left(k^{d}+k^{i}\right) p_{H 2} n_{e}
$$

Here $p_{H 2}$ is the partial pressure of $\mathrm{H}_{2}$ (mbar), $Q_{s v, H 2}$ and $Q_{s w, H 2}$ are the neutral gas sources, respectively the external injection and the wall release (mbar.l/s), $V$ is the volume of the vessel (l), $\mathrm{S}$ is the machine pumping speed (l/s, approx. equal for $\mathrm{H}_{2}, \mathrm{HD}$ and $\left.\mathrm{D}_{2}\right), k^{i}$ and $k^{d}$ are the ionization rate and dissociation rate $\left(\mathrm{cm}^{3} / \mathrm{s}\right.$, approx. equal for $\mathrm{H}_{2}, \mathrm{HD}$ and $\left.\mathrm{D}_{2}\right)$ and $n_{e}$ is the electron density $\left(\mathrm{cm}^{-3}\right)$. The recombination rate, being very low, is omitted in eq. (1) which allows writing it in the same form for $\mathrm{HD}$ and $\mathrm{D}_{2}$. Also the direct loss of neutral $\mathrm{H}_{2}$ molecules, having a temperature in the range of the gas injection balloon $(\sim 300 \mathrm{~K})$ and wall ( $\sim 40 \mathrm{~K})$ temperature, to the wall is neglected in eq. (1) as they are assumed to be mainly reflected on the wall surface. Knowing $Q_{s v, H 2}$ from the gas injection valves $\left(Q_{s v, H D}=Q_{s v, D 2}=0\right)$, the wall sources $Q_{s w, H D}$ and $Q_{s w, D 2}$ can be deduced from eq. (1) in a steady state approximation: 


$$
Q_{s w, D 2}=\left(Q_{s v, H 2}+Q_{s w, H 2}\right) \frac{p_{D 2}}{p_{H 2}}
$$

After the $\mathrm{D}_{2}$-GDC prior to the ICWC session the isotopic ratio of the wall, $D /(H+D)$, is close to 1 . From this it is expected that at the start of the ICWC session $Q_{s w, H 2}$ is very small compared to $Q_{s w, H D}$ and $Q_{s w, D 2}$, which allows to derive a meaningful lower value estimate for the release rate of $\mathrm{HD}$ and $\mathrm{D}_{2}$ in the form of eq. (3).

$$
Q_{s w, D 2} \geq Q_{s v, H 2} \frac{p_{D 2}}{p_{H 2}}
$$

Applied to the considered discharge at $t=3 \mathrm{~s}$ this leads to $Q_{s w, H D} \geq 3.3 \cdot 10^{20} \mathrm{HD} / \mathrm{s}$ and $Q_{s w, D 2} \geq 7.2 \cdot 10^{20} \mathrm{D}_{2} / \mathrm{s}$, equal to $Q_{s w, D} \geq 1.8 \cdot 10^{21} \mathrm{D} / \mathrm{s}$. The removal rate by the external pumps $Q_{p, D}$ at this same time however gives $Q_{p, D}=\left(2 p_{D 2}+p_{H D}\right) S=5.2 \cdot 10^{18} \mathrm{D} / \mathrm{s}$. The flux of particles released by the wall is thus at least 350 times higher than the flux that is actually removed from the vessel.

\section{Calculation of the total particle balance}

Ideally, in isotope exchange discharges, under saturated wall conditions, the ratio of the total amount of $\mathrm{H}$ lost to the walls, $N_{r, H}=N_{s v, H}-N_{p, H}$, and the total amount of D pumped out of the system, $N_{p, D}$, should be $N_{r, H} / N_{p, D}=1$. This ratio can be calculated from a time integration of both $Q_{s v, H 2}$ and the partial pressures multiplied by the pumping speed $S$, including discharge phase and post discharge phase. For the TEXTOR discharge under discussion this gives $N_{p, D}=2.3 \cdot 10^{20}$ and $N_{r, H}=4.5 \cdot 10^{20}$, and thus $N_{r, H} / N_{p, D} \approx 2$ meaning that for each removed $\mathrm{D}$ atom almost $2 \mathrm{H}$ atoms are retained into the wall. From this total particle balance it is concluded that the total recycling coefficient $R$ of the particle flux to the wall $Q_{f t w}$, defined as $Q_{s w}=R Q_{f t w}$, during ICWC must be $R<1$.

\section{Comparison with isotope exchange by GDC}


Both on TEXTOR and on TORE SUPRA the results from ICWC are compared with isotope exchange experiments with conventional GDC (ex. TORE SUPRA, Figure 2). The walls were preloaded by a 30 min $\mathrm{D}_{2}$-GDC on TEXTOR, respectively $\mathrm{H}_{2}$-GDC on TORE SUPRA, hereafter on both machines the gas injection was changed, proceeding with $\mathrm{H}_{2}$-GDC on TEXTOR and $\mathrm{D}_{2}$-GDC on TORE SUPRA. On TEXTOR the continuous constant gas injection resulted in a baratron pressure of $p_{\text {tot }}=0.85 \mathrm{~Pa}$. On TORE SUPRA the gas injection was feedback controlled on the total pressure $p_{\text {tot }}=0.35 \mathrm{~Pa}$. From analysis of the hydrogen partial pressures after the gas injection changeover it is found that the wall isotopes are mainly released in the form of HD (Figure 2). The release of HD decreases strongly in time showing the gradual change of the isotopic ratio of the wall.

\section{Comparison of absolute removal rates}

The absolute removal rate of HD molecules 1 minute after the changeover on TEXTOR was $Q_{p, H D, G D C}=3.4 \cdot 10^{19} \mathrm{HD} / \mathrm{s}=9.7 \cdot 10^{17} \mathrm{HD} / \mathrm{sm}^{2}$, which is about 1 order of magnitude higher than the removal rate of D during ICWC on TEXTOR reported above (also taken at approx. 1 minute of accumulated RF time after the GDC). A similar comparison between GDC and ICWC is done on TORE SUPRA, respectively 15 minutes after the change over from $\mathrm{H}_{2}$ GDC to $\mathrm{D}_{2}$-GDC, and 15 minutes of accumulated RF time. The TORE SUPRA ICRF discharge is chosen from a series of $60 \mathrm{~s}$ long semi continuous ICRF discharges, operated at nominal field ( $B_{T}=3.8 \mathrm{~T}$ ) at an RF frequency of $48 \mathrm{MHz}$ and coupled power of $40 \mathrm{~kW}$. The injection of the $\mathrm{He} / \mathrm{H}_{2}$ gas mixture (40/60) was feedback controlled via the total torus pressure $p_{\text {tot }}=0.040 \mathrm{~Pa}$. The following removal rates were recorded: $Q_{p, H D, G D C}=2.7 \cdot 10^{19} \mathrm{HD} / \mathrm{s}$ $=3.8 \cdot 10^{17} \mathrm{HD} / \mathrm{sm}^{2}$ and $Q_{p, H D, I C W C}=5.3 \cdot 10^{18} \mathrm{HD} / \mathrm{s}=7.7 \cdot 10^{16} \mathrm{HD} / \mathrm{sm}^{2}$. The removal rate of $\mathrm{D}$ for GDC is here 5 times higher than for ICWC. 
It might be expected that the higher removal rates for GDC compared to ICWC are due to the approximately 1 order of magnitude higher pressure in the analysed GDC discharges than in the ICWC discharges, both in TEXTOR and TORE SUPRA. However, the different physical properties of ICWC and GDC do not allow drawing a general dependency for the pumping rates of wall desorbed species in conditioning discharges as a function of discharge gas pressure. The last term in eq. (1) indicates that the source of particles in the plasma is not only a function of the pressure, but also of the electron density and the reaction rate. Independently of this it is shown $[3,4,5]$ that the isotopic exchange efficiency in ICWC increases when increasing the partial pressure of the operating gas.

\section{Particle recycling during GDC}

The continuous constant gas injection on TEXTOR allowed a simple comparison of the neutral pressure during the $\mathrm{D}_{2}$-GDC right before the change over with the pressure in absence of a discharge, which showed that both pressures are approximately equal. For TORE SUPRA a similar result follows from the comparison of the feedback controlled gas throughput of the valves. From this follows that, based on eq. (1), during GDC (a) $Q_{s v} \approx p S$ and consequently that (b) $Q_{s w} / V \approx\left(k^{d}+k^{i}\right) p n_{e}$, on the condition that $Q_{s w}$ and $\left(k^{d}+k^{i}\right) p n_{e} V$ are not negligible to $Q_{s v}$ and $p S$. The latter is indeed the case: right after the change over the partial pressure of HD is higher than the pressure of the injected gas; $p_{\mathrm{H} 2}$ in case of TEXTOR and $p_{D 2}$ in case of TORE SUPRA. These HD molecules can only stem from plasma wall interaction, indicating that both ionization/dissociation rates, fluxes to the wall and release rates from the wall $Q_{s w}$ are not negligible compared to the gas injection rate $Q_{s v}$. From eq. (a) it follows that the net loss of hydrogen isotopes to the wall during GDC is not significant. Similarly from eq. (b) it follows that the total recycling coefficient for GDC, $R_{G D C}$, must be close to 1 . These two findings allow stating that during GDC the wall reaches a saturated 
hydrogen content level after which extra storage of hydrogen into the wall during further GDC is not significant.

\section{Synthesis}

For isotopic exchange with glow discharges it was concluded in previous paragraphs that the recycling coefficient of the wall flux is close to one and that extra storage of particles into a saturated wall (by GDC) is not significant. On the other hand it is observed that during isotope exchange experiments with ICWC in case of saturated walls still more particles are trapped into the wall than removed by the pumps. This synthesis tries to point out a possible important mechanism for explaining a high retention rate in case of isotope exchange with ICWC and at the same time low retention rates for GDC.

\section{Estimation of the reionization probability}

An explanation of the found values $\left(Q_{r, H, G D C} \approx Q_{p, D, G D C}, N_{r, H, I C W C}>N_{p, D, I C W C}\right)$ can be sought in terms of the reionization probability $f$. Equation (1) states that a molecule in the vessel will either be ionized or dissociated, or be pumped by the machine pumps. The ionization probability, or equally the reionization probability of wall desorbed molecules, is here defined as the probability that a molecule in the vessel gets ionized or dissociated. The reionization probability for the ICWC discharge on TEXTOR discussed in the previous section can be estimated at first by comparing the wall release rate of $\mathrm{D}, Q_{s w, D}$, with the removal rate of $\mathrm{D}$, $Q_{p, D}:$

$$
f_{\text {ICWC }}=\frac{Q_{s w, D}-Q_{p, D}}{Q_{s w, D}}
$$

Taking the estimated values for this shot this results in $f_{\text {ICWC }}=99.7 \%$. Secondly, the reionization probability can also be estimated from the plasma parameters [6]:

$$
f_{\text {ICWC }}=\frac{\tau_{i}^{-1}}{\tau_{e x}^{-1}+\tau_{i}^{-1}}
$$


Here $\tau_{e x}$ is the characteristic time constant for the pumping speed ( $\left.p=p_{0} e^{-t / \tau_{e x}}\right)$ and $\tau_{i}=\left[n_{e}\left(k^{d}+k^{i}\right)\right]^{-1}[6]$ is the characteristic effective time constant for dissociation $\left(k^{d}\right)$ and ionization $\left(k^{i}\right)$. With plasma parameters $n_{e}=1 \cdot 10^{17} \mathrm{~m}^{-3}$, measured by interferometry, $T_{e}=3 \mathrm{eV}$, estimated from a 0D kinetic model for hydrogen plasmas [7], and effective reaction rates $k^{d}=1.09 \cdot 10^{-9} \mathrm{~cm}^{3} / \mathrm{s}$ and $k^{i}=4.9 \cdot 10^{-11} \mathrm{~cm}^{3} / \mathrm{s}$ calculated using a collisional-radiative model for molecular $\mathrm{H}_{2}$ [8] based on Yacora [9], this results in $f_{\text {ICWC }}=99.8 \%$, in good agreement with the previously calculated value.

In a similar way as for ICWC, a reionization probability for GD plasmas can be estimated from typical GD plasma parameters (eq. 5): $n_{e}=10^{14} / \mathrm{m}^{3}$ and $T_{e}=10 \mathrm{eV}$ [2]. The significantly lower density in GD plasmas directly leads to a much lower reionization probability $f_{G D C} \leq 86 \%$. This value is an upper limit since the electron temperature, measured by Langmuir probes, is expected to be overestimated.

\section{Estimation of total retention rates}

In the following an isotopic exchange experiment with conditioning discharges in $\mathrm{H}_{2}$ and a D saturated wall is considered. The reionization probability $f$ relates then per definition the wall desorption rate of $\mathrm{D}, Q_{s w, D}$, with the removal rate of $\mathrm{D}$ by the pumps via $Q_{p, D}=(1-f) Q_{s w, D}$, since the only source of $\mathrm{D}$ is the wall. If the isotopic ratio of the wall $D /(H+D)=1$, then the desorption rate of $\mathrm{D}$ is also related to the total flux of particles to the wall, $Q_{f t w}$, via the total recycling coefficient $R$. The outpumped flux of D can thus be written as a function of the total particle flux to the wall:

$$
Q_{p, D}=(1-f) R Q_{f t w}
$$

For the sake of comparison it is now assumed that the recycling coefficient for ICWC is as in the case of GDC close to 1 , e.g. $R_{I C W C}=R_{G D C}=0.998$. Using the estimated reionization 
probabilities and the measured removal rates of $\mathrm{D}, Q_{p, D}$, it is possible to estimate equally the required flux of particles to the walls, $Q_{f t w}$, with eq. (6), as well as the related total extra retention rate, $Q_{e r}$. The total extra retention rate is defined as $Q_{e r}=(1-R) Q_{f t w}$ and in principle disregards whether the incoming or outgoing particle consists of D or $\mathrm{H}$. The results of these estimations are summarized in Table 1.

TABLE 1. Overview estimated values for reionization probability $f$, pumping rate of $\mathrm{D} Q_{p, D}\left(\mathrm{~s}^{-1}\right)$, total wall flux $Q_{f w w}\left(\mathrm{~s}^{-1}\right)$, total extra retention rate $Q_{e r}\left(\mathrm{~s}^{-1}\right)$ for the analysed ICWC and GDC discharges.

\begin{tabular}{llllll}
\hline & $f$ & $Q_{p, D}$ & $Q_{f t w}$ & $Q_{e r}$ & $Q_{\text {er }} / Q_{p, D}$ \\
& & & & & \\
\hline GDC & .86 & $3.4 \cdot 10^{19}$ & $2 \cdot 10^{20}$ & $4 \cdot 10^{17}$ & 0.01 \\
ICWC & .998 & $5.2 \cdot 10^{18}$ & $3 \cdot 10^{21}$ & $6 \cdot 10^{18}$ & 1.2 \\
\hline
\end{tabular}

It is found that even if a small percentage of the flux of particles to the wall is permanently retained into the wall, in the considered example $(1-R)=0.2 \%$, the total retention rate during the discharge can be of the same order as the removal rate by the pumps when the particle flux to the wall is high enough, as in the case of ICWC.

\section{Optimization of ICWC efficiency}

In recent experiments on TORE SUPRA it is shown that the ratio of the total amount of $\mathrm{H}$ which is retained into the wall to the total amount of removed $\mathrm{D}$ can be brought close to 1 by optimizing the duty cycle of the RF pulses, as illustrated on Figure 3. The data points on this figure are taken from two subsequent multi-pulse $\mathrm{H}_{2}$-ICWC discharges where the wall was preloaded by $\mathrm{D}_{2}$-GDC. The $\mathrm{H}_{2}$ injection was continuous constant with $p_{\text {tot }}=20 \cdot 10^{-3} \mathrm{~Pa}$. The RF power at the generator was $60 \mathrm{~kW}$ at a frequency of $48 \mathrm{MHz}$ and the toroidal magnetic 
field was respectively 3.8T and 3.2T. Figure 3a shows that shorter RF pulses (ex 2s RF on + 38s RF off) limit the amount of retained $\mathrm{H}$ with respect to longer pulses (ex $5 \mathrm{~s}$ RF on $+35 \mathrm{~s}$ RF off and 10s RF on + 30s RF off) without severely lowering the amount of out pumped D. This can be understood as a combination of 2 effects: first because the particle flux to the wall, and thus also particle retention, is only present during the RF on time. And secondly, as indicated by the increase in gas pressure after the RF discharge in Figure 1, the release of particles from the wall continues after the RF pulse, with a characteristic time scale depending on the physical process [10]. A ratio of retained $\mathrm{H}$ over pumped $\mathrm{D}$ equal to 1 , as in the case of GDC, was also achieved with ICWC (Figure 3b).

\section{Conclusion}

In this paper the isotope exchange efficiencies of ICWC and GDC are compared by using mass spectrometry data, gas injection rates and analysing the particle balance equation of neutral hydrogen molecules in the vessel. GDC has in this analysis (5-10 times) higher removal rates of wall desorbed species by the pumps than ICWC, although the wall release rate of particles is about 10 times higher in ICWC than in GDC. The higher reionization probability in ICWC, mainly because of the higher electron density, leads to a lower removal efficiency by the external pumps of wall desorbed species than GDC. Also the measured high retention during the ICWC discharge can be understood as an effect of the high reionization probability. Optimization of the RF duty cycle can significantly improve the ratio of retained over recovered particles, without severely lowering the total amount of removed particles, bringing the efficiency of ICWC closer to that of GDC.

\section{References}


[1] H. F. Dylla, J. Vac. Sci. Technol. A6 (1988) 1276-1287

[2] H. Amemiya, et al., Japanese Journal of Applied Physics 26 (1987) 1534-1543

[3] D. Douai, et al., 12th ITPA SOL/divertor physics meeting, Amsterdam, 5-8 May 2009

[4] D. Douai, et al., This conference (2010)

[5] T. Wauters, et al., AIP Conf. Proc. 1187 (2009) 173-176

[6] E. de la Cal, and E. Gauthier, Plasma Phys. Control. Fusion 47 (2005) 197-218

[7] J. Buermans and T. Matthys, Diploma Thesis, ERM/KMS, Brussels (2006)

[8] Private communication, D. Wünderlich, March 2010

[9] D. Wünderlich, S. Dietrich, U. Fantz, JQSRT 110 (2009) 62-71

[10] V. Philipps, and J. Ehrenberg, J. Vac. Sci. Technol. 11 (1993) 437-445

\section{Figure captions}

\section{Figure 1}

Hydrogen partial pressures from the $5^{\text {th }} \mathrm{He} / \mathrm{H}_{2}$ ICWC discharge of a series of identical ICWC discharges after a $\mathrm{D}_{2}$-GDC. Parameters: $p_{H e}=35 \cdot 10^{-3} \mathrm{~Pa}, P_{R F, \text { coupled }}=50 \mathrm{~kW}, f_{R F}=29 \mathrm{MHz}$ and $B_{T}=1.92 \mathrm{~T}$.

Figure 2

Hydrogen partial pressures from an isotope exchange experiment by GDC on TORE SUPRA: The wall is first preloaded by $\mathrm{H}_{2}-\mathrm{GDC}$, hereafter at $t=0 \mathrm{~min}$. the discharge gas injection is changed over to $\mathrm{D}_{2}$-GDC.

Figure 3 
Isotope exchange efficiency as function of pulse length taken from two subsequent multipulse $\mathrm{H}_{2}$-ICWC discharges on TORE SUPRA, calculated separately for each duty cycle. $N_{s v, H}, N_{p, H}$ and $N_{p, D}$ are respectively the total injected $\mathrm{H}$, total pumped $\mathrm{H}$ and total pumped D over 1 duty cycle (40s). Discharge parameters: constant $\mathrm{H}_{2}$ gas injection with $p_{H_{2}}=0.02$ Pa, $P_{R F, \text { generator }}=60 \mathrm{~kW}, f_{R F}=48 \mathrm{MHz}$, duty cycle $t\left(P_{R F, \text { on }}\right) / t\left(P_{R F, \text { off }}\right)=x \mathrm{~s} /(40-x) \mathrm{s}$, $B_{T}=3.2-3.8 \mathrm{~T}$ and wall preloading by $\mathrm{D}_{2}$-GDC. 


\section{Figures}

\section{Figure 1}

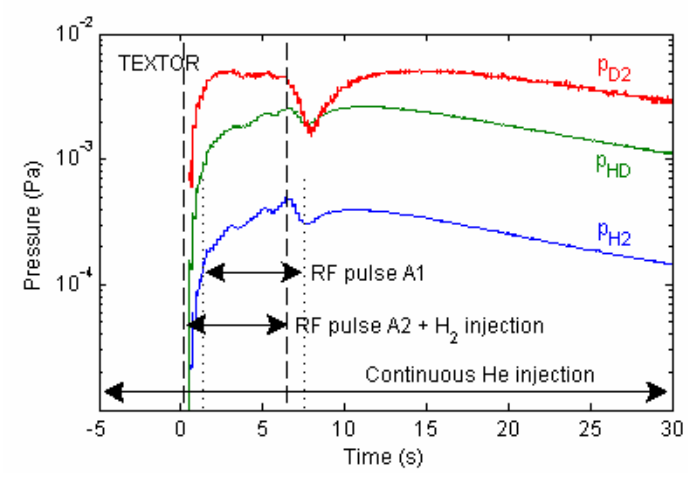


Figure 2

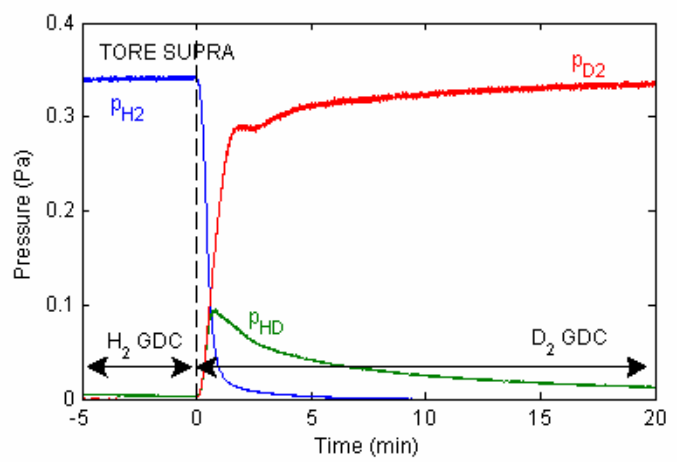

- 14 - 
Figure 3

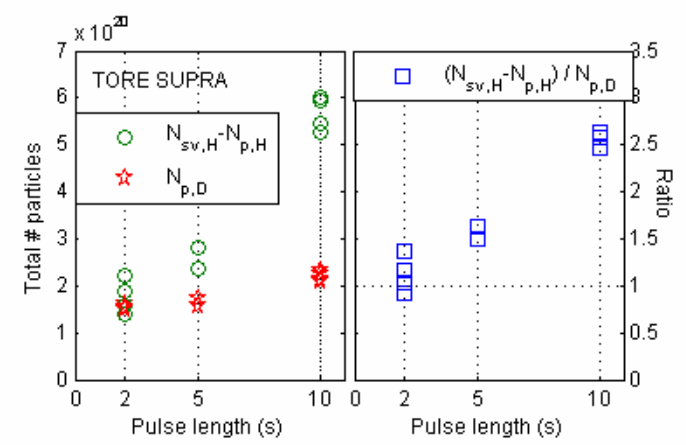

- 15 - 